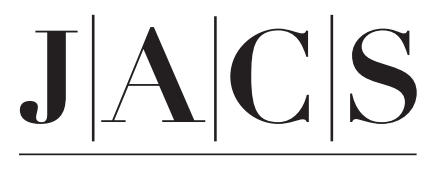

A R T I C L E S

Published on Web 04/23/2003

\title{
8-Oxoguanine Enhances Bending of DNA that Favors Binding to Glycosylases
}

\author{
John H. Miller, ${ }^{, \dagger}{ }^{\dagger}$ Cheng-Chih P. Fan-Chiang, ${ }^{\dagger}$ T. P. Straatsma, ${ }^{\ddagger}$ and \\ Michael A. Kennedy \\ Contribution from the Washington State University Tri-Cities, Richland, Washington 99352, and \\ Pacific Northwest National Laboratory, Richland, Washington 99352
}

Received November 12, 2002; E-mail: jhmiller@tricity.wsu.edu

\begin{abstract}
Molecular dynamics (MD) simulations were carried out on the DNA oligonucleotide GGGAACAACTAG:CTAGTTGTTCCC in its native form and with guanine in the central $\mathrm{G}_{19}: \mathrm{C}_{6}$ base pair replaced by 8-oxoguanine (80xoG). A box of explicit water molecules was used for solvation, and $\mathrm{Na}^{+}$counterions were added to neutralize the system. The direction and magnitude of global bending were assessed by a technique used previously to analyze simulations of DNA containing a thymine dimer. The presence of 8oxoG did not greatly affect the magnitude of DNA bending; however, bending into the major groove was significantly more probable when $80 x 0 \mathrm{G}$ replaced $\mathrm{G}_{19}$. Crystal structures of glycosylases bound to damagedDNA substrates consistently show a sharp bend into the major groove at the damage site. We conclude that changes in bending dynamics that assist the formation of this kink are a part of the mechanism by which glycosylases of the base excision repair pathway recognize the presence of 8oxoG in DNA.
\end{abstract}

\section{Introduction}

A variety of environmental and endogenous agents, including ionizing radiation and aerobic metabolism, produce oxidative DNA damage. One of the most abundant and extensively studied forms of oxidative DNA damage is 8-oxo-7,8dihydroguanine (8oxoG). ${ }^{1}$ Its tendency to mispair with adenine during replication makes $80 x \mathrm{x}$ a direct source of GC to TA transversion mutations; consequently, elaborate defense systems to protect against the genotoxic effects of 8oxoG are found in most organisms from bacteria to humans. ${ }^{2}$ Base excision repair (BER), a multistep process that begins with glycosylase activity to remove damaged bases, is a major component of these systems. ${ }^{3,4}$

Formamidopyrimidine DNA glycosylase (Fpg) removes 8oxoG and other structurally related lesions from bacterial DNA. Fpg and endonuclease VIII (Nei) belong to a family of enzymes that differs in sequence and tertiary structure from a larger family of DNA glycosylases, the Nth family named for its prototype endonuclease III, that contains the eukaryotic 8oxoG-DNA glycosylase Ogg1. Despite these differences, recent crystal structures of these glycosylases bound to their substrates ${ }^{5-8}$ exhibit remarkable similarities.

\footnotetext{
$\dagger$ Washington State University Tri-Cities.

$\doteqdot$ Pacific Northwest National Laboratory.

(1) Burrows, C. J.; Muller, J. G. Chem. Rev. 1998, 98, 1109-1151.

(2) Lindahl, T.; Wood, R. D. Science 1999, 286, 1897-1905.

(3) McCullough, A. K.; Dodson, M. L.; Lloyd, R. S. In Annual Review of Biochemistry; Richardson, C. C., Ed.; Annual Reviews: Palo Alto, CA, 1999; Vol. 68, pp 255-285.

(4) Mol, C. D.; Parikh, S. S.; Putnam, C. D.; Lo, T. P.; Tainer, J. A. Annu. Rev. Biophys. Biomol. Struct. 1999, 28, 101-128.

(5) Bruner, S. D.; Norman, D. P. G.; Verdine, G. L. Nature 2000, 403, 859866.

(6) Norman, D. P. G.; Bruner, S. D.; Verdine, G. L. J. Am. Chem. Soc. 2001 , 123, 359-360.

(7) Fromme, J. C.; Verdine, G. L. Nat. Struct. Biol. 2002, 9, 544-552.
}

10.1021/ja029312n CCC: $\$ 25.00$ @ 2003 American Chemical Society
Both Fpg and the catalytic domain of Ogg1 bind to DNA in the minor groove and extrude the damaged base through the major groove. A sharp bend into the major groove at the damaged site $\left(75^{\circ}\right.$ for Fpg bound to an abasic site and $70^{\circ}$ for Ogg1 bound to 8 oxoG) expands the minor groove and contributes to a relatively large burial of previously exposed solvent accessible surface (25.1 and $22.7 \mathrm{~nm}^{2}$ for Fpg and Ogg1, respectively). Contacts with the backbone of the damaged strand are more numerous than those with the undamaged strand, a distinctive feature of the interaction between DNA-glycosylases and their substrates that has become the basis for several models of damage recognition. Proposed mechanisms include capture of spontaneously flipped-out bases, ${ }^{9}$ strand separation, ${ }^{10}$ assistance by accessory proteins, ${ }^{11}$ migration of base flipping, ${ }^{12}$ and damaged-strand pinching. ${ }^{13}$

Structures of oligonucleotides containing 8oxoG:C and 8oxoG:A base pairs have been determined by both $\mathrm{NMR}^{14-16}$ and $\mathrm{X}$-ray crystallography. ${ }^{17,18}$ These studies confirmed the pre-

(8) Gilboa, R.; Zharkov, D. O.; Golan, G.; et al. J. Biol. Chem. 2002, 277, 19811-19816.

(9) Panayotou, G.; Brown, T.; Barlow, T.; Pearl, L. H.; Savva, R. J. Biol. Chem 1998, 273, 45-50.

(10) Vassylyev, D. G.; Morikawa, K. Structure 1996, 4, 1381-1385

(11) Cheng, X.; Blumenthal, R. M. Structure 1996, 4, 639-645.

(12) Verdine, G. L.; Bruner, S. D. Chem. Biol. 1997, 4, 329-334.

(13) Parikh, S. S.; Putnam, C. D.; Tainer, J. A. Mutat. Res. 2000, 460, 183199.

(14) Guschlbauer, W.; Duplaa, A.-M.; Guy, A.; Teoule, R.; Fazakerley, G. V. Nucleic Acids Res. 1991, 19, 1753-1758.

(15) Kouchakdjian, M.; Bodepudi, V.; Shibutani, S.; Eisenberg, M.; Johnson, F.; Grollman, A. P.; Patel, D. J. Biochemistry 1991, 30, 1403-1412.

(16) Oda, Y.; Uesugi, S.; Ikehara, M.; Nishimura, S.; Kawase, Y.; Ishikawa, H.; Inoue, H.; Ohtsuka, E. Nucleic Acids Res. 1991, 19, 1407-1412.

(17) Lipscomb, L. A.; Peek, M. E.; Morningstar, M. L.; Verghis, S. M.; Miller, E. M.; Rich, A.; Essigmann, J. M.; Williams, L. D. Proc. Natl. Acad. Sci. U.S.A. 1995, 92, 719-723.

(18) Shui, X.; Sines, C. C.; McFail-Isom, I.; VanDerveer, D.; Williams, L. D. Biochemistry 1998, 37, 16877-16887. 
dominance of the 6,8-diketo tautomer at physiological $\mathrm{pH},{ }^{19}$ the normal anti-anti form of the 8 oxoG: $\mathrm{C}$ base pair, and the Hoogsteen syn-anti form of the 8oxoG:A. On the basis of these data, patterns of hydrogen-bond donor and acceptor groups in the grooves of DNA seemed critical for recognition of 8oxoG by DNA glycoslyases. ${ }^{20}$ Experimental studies of substrate specificity ${ }^{21}$ supported this conclusion.

Dodson and Lloyd ${ }^{22}$ applied principle-component analysis to DNA structures sampled from MD simulations of substrates of Fpg and MutY containing 8oxoG:C and 8oxoG:A, respectively. $74 \%$ of the parameters judged to be significant in discriminating substrates from undamaged DNA came from analysis of the dynamics of the oligomer containing 8oxoG:C, which indicated that this oligomer often adopted conformations conducive to Fpg binding. Most of the backbone parameters in this subset were associated with the undamaged strand, which suggested that Fpg flips out cytosine when it binds a substrate that contains an 8oxoG:C base pair.

Recent structural and biochemical studies ${ }^{23}$ have revealed the important role of kinetics in damage recognition by glycosylases. Kinetic proof reading has long been recognized as a major contributor to the fidelity of biosynthesis. ${ }^{24}$ Kinetic control of damage recognition is based on the idea that complexes between glycosylases and undamaged DNA decay too quickly to permit base flipping, while at damaged sites, some aspect of proteinDNA interaction stabilizes the complex for a longer period. The sharp bend or kink in DNA at the damaged base observed in crystal structures ${ }^{5-8}$ suggests that a reduced force constant for bending into the major groove could enhance the stability of these complexes. In addition, DNA bending lowers the free energy of base opening; ${ }^{25,26}$ hence, if a bent configuration can be maintained, then the energy barrier to base flipping remains relatively low.

Osman et al. ${ }^{27}$ proposed that changes in the bending dynamics of DNA induced by thymine dimers ${ }^{28}$ were part of the mechanism by which $\mathrm{T} 4$ endonuclease $\mathrm{V}$ recognizes these lesions. In this paper, we report calculations similar to those performed on DNA containing a thymine $\operatorname{dimer}^{28}$ to investigate the effects of 8 oxoG on the bending dynamics of a 12-basepair oligonucleotide. Larger curvature is slightly more probable when 8oxoG is present; however, the main effect is on the preferred direction of bending. In the absence of 8oxoG, bending into either groove is about equally likely; however, when $80 x 0 \mathrm{G}$ is present, bending into the major groove is strongly preferred.

\section{Methods}

2.1. Force-Field Parameters. Partial atomic charges for 8 -oxo-7,8dihydroguanine in the 6,8-diketo form were evaluated in the RESP

(19) Cho, B. P.; Kadlubar, F. F.; Culp, S. J.; Evans, F. E. Chem. Res. Toxicol. 1990, 3, 445-452.

(20) Grollman, A. P.; Johnson, F.; Tchou, J.; Eisenberg, M. In DNA Damage: Effects on DNA Structure and Protein Recognition; Wallace, S. S., van Houten, B., Kow, Y.-W., Eds.; Annals NY Acad. Sci.: New York, 1994; Vol. 726, pp 208-214.

(21) Tchou, J.; Bodepudi, V.; Shibutani, S.; Antoshechkin, I.; Miller, J.; Grollman, A. P.; Johnson, F. J. Biol. Chem. 1994, 269, 15318-15324.

(22) Dodson, M. L.; Lloyd, R. S. Mutat. Res. 2001, 487, 93-108.

(23) Tainer, J. A. Prog. Nucleic Acid Res. 2001, 68, 299-304.

(24) Hopfield, J. J. Proc. Natl. Acad. Sci. U.S.A. 1974, 71, 4135-4139.

(25) Ramstein, J.; Lavery, R. Proc. Natl. Acad. Sci. U.S.A. 1988, 85, 72317235 .

(26) van Aalten, D. M. F.; Erlanson, D. A.; Verdine, G. L.; Joshua-Tor, L. Proc. Natl. Acad. Sci. U.S.A. 1999, 96, 11809-11814

(27) Osman, R.; Fuxreiter, M.; Luo, N. Comput. Chem. 2000, 24, 331-339.

(28) Miaskiewicz, K.; Miller, J.; Cooney, M.; Osman, R. J. Am. Chem. Soc. 1996, 118, 9156-9163.
Table 1. Existing AMBER Force-Field Parameters Substituted for Parameters Needed To Simulate DNA Containing 80xoG

\begin{tabular}{ll}
\hline missing parameter & existing AMBER parameter \\
\hline $\mathrm{CK}-\mathrm{O}$ & $\mathrm{C}-\mathrm{O}$ \\
$\mathrm{CB}-\mathrm{NA}$ & $\mathrm{CB}-\mathrm{NB}$ \\
$\mathrm{CK}-\mathrm{NA}$ & $\mathrm{CK}-\mathrm{NB}$ \\
$\mathrm{CB}-\mathrm{CB}-\mathrm{NA}$ & $\mathrm{CB}-\mathrm{C}-\mathrm{NA}$ \\
$\mathrm{C}-\mathrm{CB}-\mathrm{NA}$ & $\mathrm{C}-\mathrm{CB}-\mathrm{NB}$ \\
$\mathrm{CB}-\mathrm{NA}-\mathrm{H}$ & $\mathrm{CC} / \mathrm{CR} / \mathrm{CW}-\mathrm{NA}-\mathrm{H}$ \\
$\mathrm{CB}-\mathrm{NA}-\mathrm{CK}$ & $\mathrm{CB}-\mathrm{NB}-\mathrm{CK}$ \\
$\mathrm{NA}-\mathrm{CK}-\mathrm{O}$ & $\mathrm{NA}-\mathrm{C}-\mathrm{O}$ \\
$\mathrm{N}^{*}-\mathrm{CK}-\mathrm{O}$ & $\mathrm{N} *-\mathrm{C}-\mathrm{O}$ \\
$\mathrm{N}^{*}-\mathrm{CK}-\mathrm{NA}$ & $\mathrm{N} *-\mathrm{C}-\mathrm{NA}$ \\
$\mathrm{CK}-\mathrm{NA}-\mathrm{H}$ & $\mathrm{CC} / \mathrm{CR} / \mathrm{CW}-\mathrm{NA}-\mathrm{H}$ \\
$\mathrm{X}-\mathrm{CB}-\mathrm{NA}-\mathrm{X}$ & $\mathrm{X}-\mathrm{CB}-\mathrm{N} *-\mathrm{X}$ \\
$\mathrm{X}-\mathrm{CK}-\mathrm{NA}-\mathrm{X}$ & $\mathrm{X}-\mathrm{CC}-\mathrm{NA}-\mathrm{X}$ \\
$\mathrm{X}-\mathrm{X}-\mathrm{CK}-\mathrm{O}$ & $\mathrm{X}-\mathrm{X}-\mathrm{C}-\mathrm{O}$ \\
& \\
\hline
\end{tabular}

model of Bayly et al. ${ }^{29}$ based on an electrostatic potential energy calculated with the 6-31G* basis set for consistency with other atomic charges in the AMBER force field. ${ }^{30}$ The total charge on a methyl group added at $\mathrm{N} 9$ to replace the sugar moiety was constrained at $0.0888 \mathrm{eu}$, which made the net charge on 8 oxoG the same as that on guanine in the AMBER force field. ${ }^{30}$ The resulting atomic charges are as follows: $\mathrm{N} 1(-0.4025), \mathrm{H} 1(0.3266), \mathrm{C} 2(0.7208), \mathrm{N} 2(-0.9625), 1 \mathrm{H} 2(0.4371)$, $2 \mathrm{H} 2(0.4371), \mathrm{N} 3(-0.6118), \mathrm{C} 4(0.2108), \mathrm{C} 5(-0.0211), \mathrm{C} 6(0.4299), \mathrm{O6}-$ $(-0.55), \mathrm{N} 7(-0.5129), \mathrm{H} 7(0.4077), \mathrm{C} 8(0.4468), \mathrm{O} 8(-0.5558)$, and N9(0.111).

Carbon and nitrogen atoms on the five-membered ring of guanine are not normally bonded to extracyclic oxygen and hydrogen atoms, respectively; hence, 8oxoG introduces a need for new AMBER forcefield parameters. Extracyclic $\mathrm{O}$ and $\mathrm{H}$ are bound to $\mathrm{C}$ and $\mathrm{N}$, respectively, on the six-membered ring of guanine. We assumed that force-field parameters that apply to these interactions in the sixmembered ring are a reasonable approximation to their appearance in the five-membered ring of $80 x o G$. The resulting connection to existing AMBER force-field parameters is shown in Table 1.

2.2. Base Sequence and Starting Structures. The sequence con-

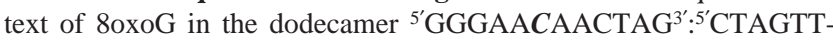
$80 \boldsymbol{G}$ TTCCC ${ }^{3^{\prime}}$ has the form shown by Hatahet et al ${ }^{31}$ to be cleaved efficiently by Fpg. Canonical B-DNA ${ }^{32}$ was used as a starting structure for all MD simulations when native guanine was present in the $\mathrm{C}_{6}: \mathrm{G}_{19}$ base pair. One starting structure for a 2 ns simulation of the damaged oligomer was obtained from canonical B-DNA by replacing the hydrogen at the $\mathrm{C} 8$ position on $\mathrm{G}_{19}$ with an oxygen atom and adding hydrogen to the N7 position. Another 2 ns simulation of the damaged oligomer was performed after analysis of the first simulation suggested an effect of $80 x o G$ on DNA bending dynamics. The starting structure for the second simulation was obtained by making the above modifications at $\mathrm{C} 8$ and $\mathrm{N} 7$ on $\mathrm{G}_{19}$ in a conformation of the native sequence that was bent into the minor groove; hence, the second MD simulation of the damaged oligomer was a test of the dependence on the starting structure of our observation that $80 x o G$ induces a preference for bending into the major groove.

To neutralize the net charge on the 12-base-pair DNA oligonucleotide, $22 \mathrm{Na}^{+}$counterions were added at energetically favorable positions at least $1 \mathrm{~nm}$ from any DNA atom. DNA and counterions were placed in a box of SPC/E water molecules. ${ }^{33}$ The total system

(29) Bayly, C.; Cieplak, P.; Cornell, W.; Kollman, P. A. J. Phys. Chem. 1993, 97, 10269-10280.

(30) Cornell, W. D.; Cieplik, P.; Bayly, C. I.; et al. J. Am. Chem. Soc. 1995, 117, 5179-5197.

(31) Hatahet, Z.; Meixia, Z.; Reha-Krantz, L. J.; Morrical, S. W.; Wallace, S. S. Proc. Natl. Acad. Sci. U.S.A. 1998, 95, 8556-8561.

(32) Arnott, S.; Chandrasekaran, R.; Birdsall, D. L.; Leslie, A. G. W.; Ratliff, R. L. Nature 1980, 283, 743-746.

(33) Berendsen, H. J. C.; Grigera, J. R.; Straatsma, T. P. J. Phys. Chem. 1987, $91,6269-6271$. 
contained 760 DNA atoms (761 when 8 oxoG replaced $\mathrm{G}_{19}$ ), 22 counterions, and 6966 water molecules for a total of 21680 atoms.

2.3. Equilibration and Data Collection. All MD simulations were conducted with the NWChem ${ }^{34}$ computational chemistry package using the PARM99 version ${ }^{35}$ of the AMBER force field. ${ }^{30}$ A short-range cutoff of $1 \mathrm{~nm}$ was placed on nonbonded interactions. Long-range electrostatic interactions were treated by the particle-mesh Ewald (PME) method ${ }^{36}$ using a $64 \times 64 \times 64$ grid. Bond lengths between hydrogen and heavy atoms were constrained by the SHAKE algorithm ${ }^{37}$ with a tolerance of $10^{-4} \mathrm{~nm}$. To equilibrate the solvent, $20 \mathrm{ps}$ of MD simulation at 50 K were followed by 10 ps at $298 \mathrm{~K}$, both with solute atoms fixed. Velocities were reassigned every 0.2 ps. To equilibrate the solute, 200 steps of steepest descent energy minimization were followed by successive 20 ps simulations at 50,100, 150, 200, and $298 \mathrm{~K}$, all with solvent coordinates fixed. After equilibration, 2 ns of MD simulation was performed at constant temperature and pressure without constraints on either solute or solvent. Temperature was maintained at $298 \mathrm{~K}$ by coupling to a Berendsen thermostat ${ }^{38}$ with a relaxation time of $0.1 \mathrm{ps}$. A 2 fs time step was used in all MD simulations. Physical properties (temperature, pressure, density, total energy, etc.) were recorded every $0.2 \mathrm{ps}$ along with the coordinates of solute atoms and counterions. The coordinates of all atoms, including water molecules, were stored every 2 ps.

2.4. Analysis. The $\mathrm{NWChem}^{34}$ analysis package was used to display root-mean-square deviations (RMSDs) from various reference structures as well as time series of system properties and selected interatomic distances. The CURVES algorithm ${ }^{39}$ was applied as a standalone version and as implemented in the Dials and Windows program ${ }^{40}$ to extract helical parameters from the Cartesian coordinates of solute atoms. Global DNA curvature was assessed by a method described previously ${ }^{28}$ that involves fitting a circle to the CURVES helical axis reference points ${ }^{39}$ projected onto the best plane passing through them. ${ }^{41}$ The reciprocal of the radius of curvature was chosen as the measure of bending magnitude. To specify the direction of bending, a vector from the helical axis reference point of the $\mathrm{C}_{6}: \mathrm{G}_{19}$ base pair to the center of curvature was projected onto the best plane through the atoms of the $\mathrm{C}_{6}: \mathrm{G}_{19}$ base pair. The case where the projected vector coincided with the pseudo-dyad axis ${ }^{42}$ and pointed into the major groove was chosen as a bending direction of $0^{\circ}$.

\section{Results and Discussion}

Figure 1 shows root-mean-square deviations (RMSDs) from ideal A- and B-DNA of simulated structures with and without 8oxoG present. Both MD simulations, which started from B-DNA conformations, show the development of A-DNA-like properties during the first $200 \mathrm{ps}$. The native sequence continues this development between 400 and 800 ps and then relaxes back to conformations with RMSDs from B-DNA in the $0.25-0.35$ $\mathrm{nm}$ range. When $80 x \mathrm{GG}$ is present, RMSDs from B-DNA stay in the $0.2-0.3 \mathrm{~nm}$ range from 0.2 to $2 \mathrm{~ns}$ except for a $200 \mathrm{ps}$ period around $1 \mathrm{~ns}$ in which conformations become slightly more like A-DNA.

(34) Harrison, R. J.; Nichols, J. A.; Straatsma, T. P.; et al. NWChem, a computational chemistry package for parallel computers, Version 4.0. Richland, WA: Pacific Northwest National Laboratory, 2000.

(35) Cheatham, T. E.; Cieplak, P.; Kollman, P. A. J. Biomol. Struct. Dyn. 1999, $16,845-862$.

(36) Essmann, U.; Perera, L.; Berkowitz, M. J. Chem. Phys. 1995, 103, 85778593

(37) Ryckaert, J. P.; Ciccotti, G.; Berendsen, H. J. C. J. Comput. Phys. 1977 $23,327-341$

(38) Berendsen, H. J. C.; Postma, J. P. M.; van Gunsteren, W. F.; DiNola, A. Haak, J. R. J. Chem Phys 1984, 81, 3684-3690.

(39) Lavery, R.; Sklenar, H. J. Biomol. Struct. Dyn. 1989, 6, 655-667.

(40) Ravishanker, G.; Swaminatham, S.; Beveridge, D. L.; Lavery, R.; Sklenar, H. J. Biomol. Struct. Dyn. 1989, 6, 669-699.

(41) Soumpasis, D. M.; Tung, C.-S.; Garcia, A. E. J. Biomol. Struct. Dyn. 1991, 8, 867-888

(42) Saenger, W. Principles of Nucleic Acid Structure; Springer-Verlag: New York, 1983; p 123.
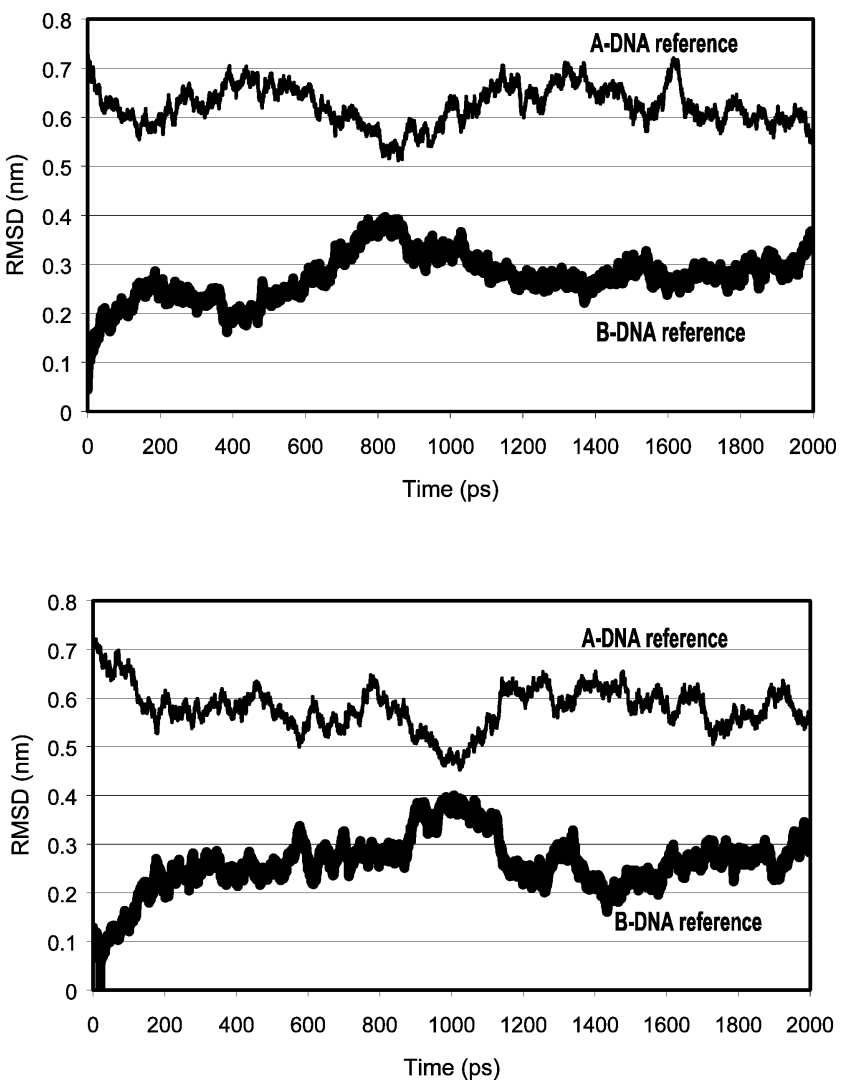

Figure 1. RMSD from ideal A- and B-DNA during 2 ns MD simulations of oligonucleotide GGGAACAACTAG:CTAGTTGTTCCC; native form (top), G19 replaced by $80 x 0 \mathrm{G}$ (bottom)

Most of the base pairs in both the native and the damaged oligomers exhibited values of the CURVES ${ }^{39}$ parameters $x$-displacement (XDP) and inclination (INC) closer to B-DNA values $\left(-0.07 \mathrm{~nm}\right.$ and $\left.-5.9^{\circ}\right)$ than to A-DNA values $(-0.54 \mathrm{~nm}$ and $\left.19^{\circ}\right)$. Base pairs near the end of the oligonucleotide with 5 purine bases in the same strand showed large INC like that seen in A-DNA. Backbone torsion angles also showed a preference for B-DNA-like values. Most of the exceptions to this general characteristic were seen in the sugar pucker and $\delta$ torsion angle at $\mathrm{C}_{6}$, the base opposite 8 oxoG in the damaged oligomer.

No evidence for opening of the 8oxoG:C base pair was seen in either of the $2 \mathrm{~ns}$ MD simulations carried out on the damaged oligomer. This is consistent with other recent simulations of DNA oligomers containing $80 \mathrm{ooG}^{22,43}$ as well as experimental studies $^{44}$ that established a millisecond time scale for base flipping. Ishida ${ }^{43}$ observed BI to BII transitions in the $\epsilon-\xi$ backbone torsion angles in an MD simulation of the $12 \mathrm{mer}$ d(CGCGAATTCGCG) $)_{2}$ with 8oxoG replacing $\mathrm{G}_{4}$. We found these transitions at $\mathrm{C}_{6}$ in our simulations when 8oxoG was present in the opposite strand, but they were not present in the backbone of the damaged strand.

Unlike Ishida, ${ }^{43}$ we did not find any significant deviations of 8oxoG's glycosyl angle, XPD of the 8oxoG:C base pair, or twist of the double helix from values expected for B-DNA. ${ }^{39}$ These differences in simulation results are probably due to the differences in base sequence and force-field parameters. Ishida used the force field reported by Cornell et al. ${ }^{30}$ and allowed the

(43) Ishida, H. J. Biomol. Struct. Dyn. 2002, 19, 839-851.

(44) Stivers, J. T. Biochemistry 1999, 38, 952-963. 


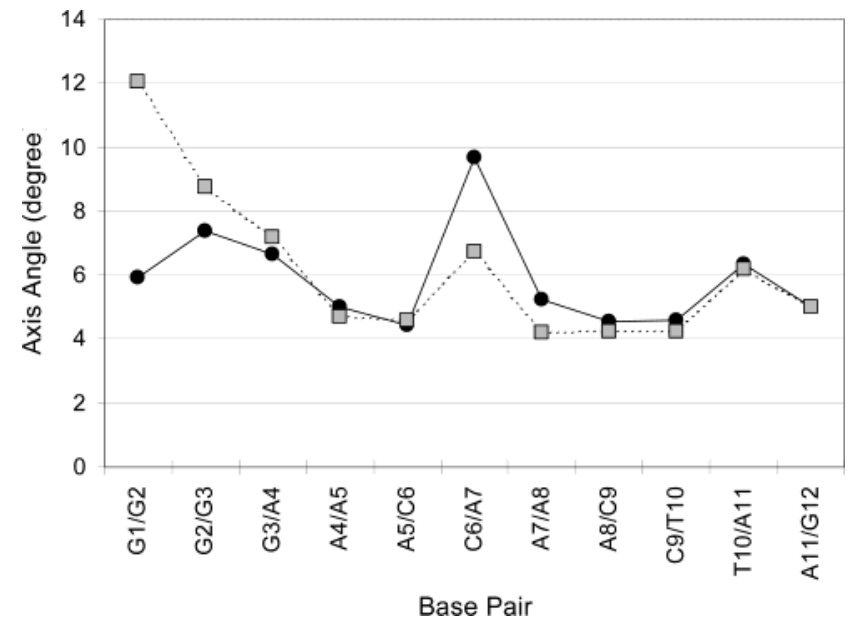

Figure 2. Mean axis kinks at base pairs in GGGAACAACTAG: CTAGTTGTTCCC from $2 \mathrm{~ns}$ simulations of the native form (ם) and with $\mathrm{G}_{19}$ replaced by $80 x o \mathrm{G}(\bullet)$.

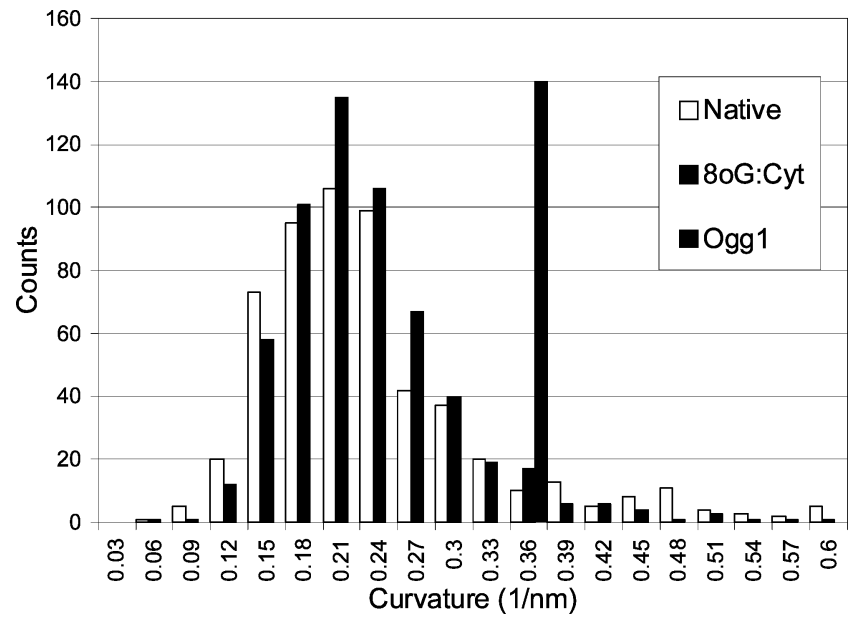

Figure 3. Distribution of global axis curvature in GGGAACAACTAG: CTAGTTGTTCCC from $2 \mathrm{~ns}$ simulations of the native form and with $\mathrm{G}_{19}$ replaced by 8 oxoG as compared to the curvature of DNA in the hOgg1 crystal structure. ${ }^{5}$

partial charge on the $\mathrm{O}^{\prime}$ atom of the damaged deoxyribonucleoside to be $27 \%$ more negative than the standard AMBER value. Even though the partial charge on the $\mathrm{O} 8$ atom of $80 x \mathrm{oG}$ was essentially the same as that in our model, more electrostatic repulsion between $\mathrm{O}^{\prime}$ and $\mathrm{O} 8$ was present in Ishida's simulation of the damaged oligomer.

Average values of the axis-angle CURVES parameter ${ }^{39}$ from $2 \mathrm{~ns}$ simulations in the presence and absence of 8oxoG are shown in Figure 2. Standard deviations of the angles between segments of the helical axis are typically $2-4^{\circ}$ in our simulations; hence, the increase in the kink of the helical axis between the $\mathrm{C}_{6}: \mathrm{G}_{19}$ and $\mathrm{A}_{7}: \mathrm{T}_{18}$ base pairs in the presence of 8oxoG is not statistically significant. Nevertheless, the trend suggests that 8oxoG influences bending into the major groove. To further investigate this effect, the magnitude and direction of global bending were analyzed by a method used previously for DNA containing a thymine $\operatorname{dimer}^{28}$ (see the Methods section). Qualitatively similar results were obtained with the Madbend analysis program ${ }^{45}$ where global bending is assessed by accumulated roll and tilt of base pairs.

The presence of 8oxoG had very little effect on the magnitude of bending. A small shift toward larger curvature is evident in
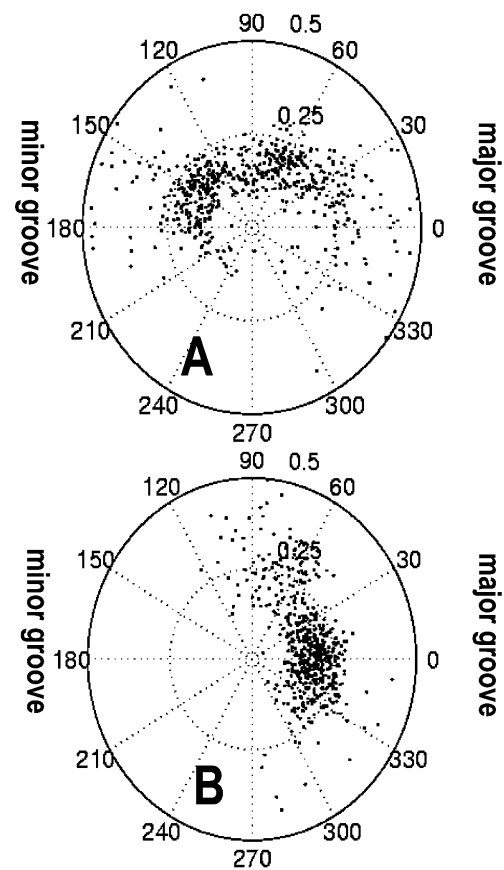

공.

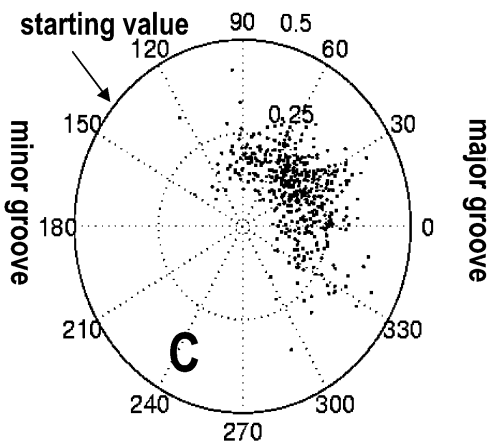

Figure 4. Polar plots of curvature $\left(\mathrm{nm}^{-1}\right)$ versus direction of bending for conformations sampled from 2 ns MD simulations of GGGAACAACTAG: CTAGTTGTTCCC in the native form (A), with 8oxoG replacing $\mathrm{G}_{19}$ in an ideal B-DNA starting structure (B), and with 8oxoG replacing $\mathrm{G}_{19}$ in a starting structure bent toward the minor groove $(C)$. Bending characteristics plotted in $(\mathrm{C})$ are from the second nanosecond of MD simulation.

the distribution of bending magnitudes shown in Figure 3. The conformation of DNA in the crystal structure $^{5}$ of Ogg1 bound to a substrate with $80 x o G$ flipped out exhibits more of a kink than the smooth bending typical of our simulations; nevertheless, application of our bending analysis to those DNA coordinates gave a magnitude of bending twice as large as the average curvature found in our simulations. Results shown in Figure 3 indicate that this magnitude of bending rarely occurs in the absence of protein binding.

The curvature of DNA conformations sampled from our MD simulations is plotted as a function of their direction of bending in Figure 4 with bending directly into the major and minor grooves denoted by $0^{\circ}$ and $180^{\circ}$, respectively. Effects of 8oxoG on the preferred direction of bending are clearly evident. Conformations sampled from the $2 \mathrm{~ns}$ simulation of the native sequence (A) exhibit a slight preference for bending toward the minor groove. Samples from our first simulation of the damaged oligomer (B) showed a strong preference for bending into the major groove.

(45) Strahs, D.; Schlick, T. J. Mol. Biol. 2000, 301, 643-663. 


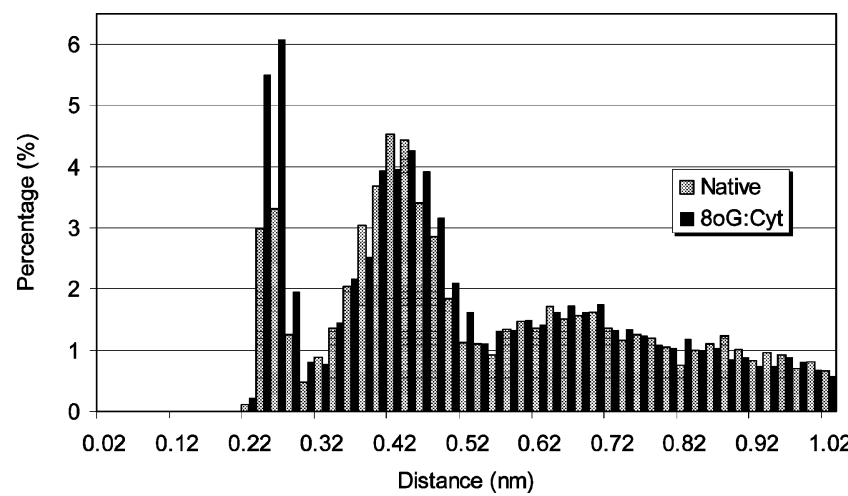

Figure 5. Distribution of minimum distances between $\mathrm{Na}^{+}$counterions and an atom of GGGAACAACTAG:CTAGTTGTTCCC during 2 ns MD simulations of the native form and with $\mathrm{G}_{19}$ replaced by 8oxoG.

To ensure that this preference was not an artifact of the initial ideal B-DNA starting structure, a second 2 ns simulation of the damaged oligomer was started from a conformation bent in the preferred direction of the native sequence $\left(140^{\circ}\right)$ and with a curvature near the mean value of $0.2 \mathrm{~nm}^{-1}$ seen in all simulations. During the first nanosecond of this simulation, a population of conformations bent into the major groove gradually emerged. Global bending characteristics of samples from the second nanosecond of this simulation of the damaged oligomer (C) also exhibit a strong preference for bending into the major groove.

Preferential bending of DNA has been associated with asymmetric phosphate neutralization. ${ }^{46}$ To investigate whether this is the mechanism for the changes in bending dynamics induced by 8 oxoG, we analyzed the effect of 8 oxoG on the spatial distribution of counterions in our simulations. The minimum distance of each counterion from an atom of the DNA oligonucleotide was determined in conformations sampled from MD trajectories. Figure 5 shows a histogram of these distances with the fraction of counterions found at a given distance from DNA plotted on the vertical axis.

The following groups are evident in Figure 5: (1) counterions less than $0.32 \mathrm{~nm}$ from a DNA atom, (2) counterions with a broader distribution of separations from DNA and a mean value of about $0.45 \mathrm{~nm}$, and (3) counterions with a significant probability of being far from DNA (exceeding $2 \mathrm{~nm}$ in some cases) but with a most probable separation of about $0.7 \mathrm{~nm}$. In simulations of the native sequence, the mean number of counterions in each of these groups was 2.1, 6.5, and 13.4, respectively. When 8 oxoG was present, these populations changed to $3.5,6.6$, and 11.9 , respectively.

The presence of $80 x o G$ caused counterions to stay closer to DNA with the major change being the number of counterions in close proximity to a DNA atom. These close contacts were not uniformly distributed. A counterion near the $\mathrm{O} 2$ atom of base $\mathrm{T}_{18}$ accounted for $21 \%$ of the cases where a counterion was within $0.32 \mathrm{~nm}$ of a DNA atom (first group defined above). Counterions near the $\mathrm{O} 8$ atom of $80 x \mathrm{oG}$ and phosphate oxygen atoms adjacent to the $8 \mathrm{oxoG}_{19}: \mathrm{C}_{6}$ base pair accounted for another $30 \%$ of the population of this group. None of these atoms showed a particular attraction for counterions in simulations of the native sequence.

Greater affinity of counterions for oxygen atoms in or near the damaged base is consistent with the changed electrostatic potential due to the presence of 8 oxoG.${ }^{47}$ However, the $\mathrm{O} 2$ atom

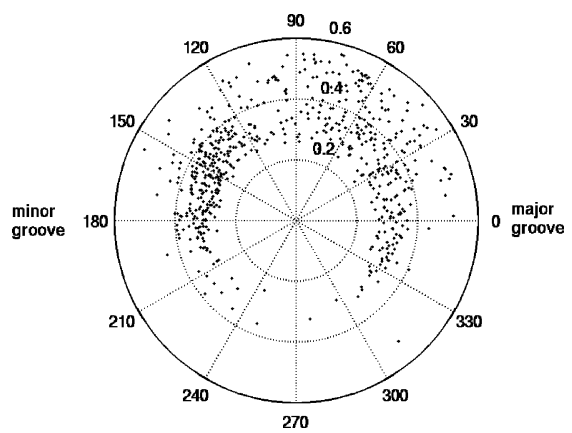

Figure 6. Polar plot of the distance (nm) between atoms $\mathrm{H} 8$ and $\mathrm{O} 2 \mathrm{P}$ in nucleotide $\mathrm{G}_{19}$ of GGGAACAACTAG:CTAGTTGTTCCC as a function of global bending direction during a $2 \mathrm{~ns}$ MD simulation of the native oligonucleotide.

of $\mathrm{T}_{18}$ is on the minor-groove side of the $\mathrm{T}_{18}: \mathrm{A}_{7}$ base pair, and close contacts between counterions and oxygen atoms of the 8 oxoG $_{19}: \mathrm{C}_{6}$ base pair do not preferentially neutralize the damaged strand. Hence, asymmetric neutralization does not appear to be the mechanism for enhanced bending into the major groove when 8oxoG is present in DNA.

Electrostatic repulsion between the $\mathrm{O} 8$ atom of 8oxoG and nearby oxygen atoms of the DNA backbone is also a possible mechanism for preferred bending of the damaged oligonucleotide into the major groove. $\mathrm{H} 8$ to $\mathrm{O} 2 \mathrm{P}$ separations in the $\mathrm{G}_{19}$ nucleotide of the native oligomer are plotted as a function of bending direction in Figure 6.

Figure 4A showed that the native oligomer frequently had bending directions between $120^{\circ}$ and $180^{\circ}$. That cluster of samples from the $2 \mathrm{~ns}$ simulation of the native oligomer is also evident in Figure 6 and is associated with H8-O2P separations that are rarely larger than $0.4 \mathrm{~nm}$. Figure 4A also showed a cluster of conformations of the undamaged oligomer with bending directions centered about $60^{\circ}$. These conformations have a broader distribution of distances between $\mathrm{H} 8$ and $\mathrm{O} 2 \mathrm{P}$ with a significant probability of separations as large as 0.55 nm (see Figure 6).

The correlation coefficient between bending direction and the separation of $\mathrm{H} 8$ and $\mathrm{O} 2 \mathrm{P}$ is -0.227 for the 503 points shown in Figure 6 between $0^{\circ}$ and $180^{\circ}$, which suggests that the $\mathrm{H} 8$ atom of $\mathrm{G}_{19}$ is closer to the backbone phosphate group when the native oligonucleotide is bent toward the minor groove. A similar result was obtained for the correlation between bending directions and $\mathrm{H} 8$ to $\mathrm{O} 1 \mathrm{P}$ distances (correlation coefficient $=$ $-0.255)$. A weaker correlation exists between bending directions and $\mathrm{H} 8$ to $\mathrm{O}^{\prime}$ ' distances (correlation coefficient $=0.152$ ). For the native sequence, bending toward the minor groove correlates with shorter $\mathrm{H} 8-\mathrm{O} 2 \mathrm{P}$ and $\mathrm{H} 8-\mathrm{O} 1 \mathrm{P}$ separations; therefore, electrostatic repulsion between $\mathrm{O} 8$ and these phosphate oxygens is probably one of the reasons why bending into the minor groove is avoided when $\mathrm{G}_{19}$ is replaced by 8oxoG.

\section{Conclusions}

No tendency for base opening was detected in 2 ns MD simulations of the oligonucleotide GGGAACAACTAG: CTAGTT8oxoGTTCCC. The most pronounced local changes induced by the presence of 8oxoG were an attraction for counterions and a slightly larger kink in the helical axis between

(46) Strauss, J. K.; Maher, L. J. Science 1994, 266, 1829-1834

(47) Aida, M.; Nishimura, S. Mutat. Res. 1987, 192, 83-89. 
base pairs $80 x \mathrm{xG}_{19}: \mathrm{C}_{6}$ and $\mathrm{T}_{18}: \mathrm{A}_{7}$. The damaged base had a large effect on the dynamics of global bending. Conformations with the oligomer bent in the direction of minor groove were rarely seen when 8 oxoG was present even though that was the preferred direction of bending for the native sequence.

The mechanisms that relate local perturbations due to 8oxoG to changes in global bending dynamics are uncertain. Attraction of counterions to the damage site did not preferentially neutralize atoms on the major-groove face of the oligomer. Electrostatic repulsion between $\mathrm{O} 8$ and the phosphate oxygen atoms of the 8oxoG nucleotide appears to be one of the factors inhibiting bending into the minor groove when $80 x 0 \mathrm{G}$ is present. As Aida and Nishimura ${ }^{47}$ point out, changes in the electrostatic potential also affect stacking interactions, which may influence bending dynamics. Further investigation of these mechanisms is needed.

Because binding of Ogg1 to DNA containing 8oxoG induces a sharp bend into the major groove at the damage site, ${ }^{5}$ a preference for bending into the major groove could be a factor in recognition of 8oxoG by this DNA glycosylases, as Osman et al. ${ }^{27}$ proposed for the recognition of thymine dimers by $\mathrm{T} 4$ endonuclease $\mathrm{V}$. This concept is being investigated further by potential-of-mean-force calculations ${ }^{48}$ to determine the effect of $80 x o G$ on the free energy of conformational changes that mimic the "pinch-push-pull" mechanism of base flipping. ${ }^{13}$ These changes include decreasing the separation of phosphate groups at the damaged site and rotation of the damaged base out of the helix.

Acknowledgment. This research was supported by the Office of Science (BER), U.S. Department of Energy, Grant No. DEFG03-02ER63350. Computing resources were available through a Computational Grand Challenge Application grant from the Molecular Sciences Computing Facility in the Environmental Molecular Sciences Laboratory. The NWChem 4.0.1 computational chemistry package for massively parallel computers was used for this study. NWChem was developed by the Molecular Sciences Software group of the Pacific Northwest National Laboratory, which is operated by the Battelle Memorial Institute for the U.S. Department of Energy.

JA029312N

(48) Straatsma, T. P. In Reviews of Computational Chemistry; Lipkowitz, K. B., Boyd, D. B., Eds.; VCH Publishers: New York, 1996; Vol. 9, pp 81127. 